\title{
Therapeutic challenges of hepatic mucormycosis in a renal transplant recipient: a case report
}

\author{
Chang Hun Lee ${ }^{1}$, Cheol Woong Jung ${ }^{1}$, Jun Gyo Gwon ${ }^{1}$, Myung Gyu Kim² \\ ${ }^{1}$ Division of Transplantation Vascular Surgery, Department of Surgery, Korea University College of Medicine, Seoul, Korea \\ ${ }^{2}$ Division of Nephrology, Department of Internal Medicine, Korea University College of Medicine, Seoul, Korea
}

Background: Mucormycosis is a rare, highly lethal opportunistic fungal disease affecting immune-compromised patients. It accounts for about $2 \%$ of invasive fungal infections occurring within 1 year after solid organ transplantation. We report here a case of successful treatment for hepatic mucormycosis with abscess formation that occurred 2 months after kidney transplantation. Methods: A 59-year-old woman who underwent a deceased donor kidney transplantation 2 months ago was hospitalized with abdominal pain and fever. She had thymoglobulin induction therapy and was on conventional maintenance immunosuppressive therapy to prevent rejection. There were no specific complications except delayed graft function after kidney transplantation. The computed tomography (CT) scan was performed, demonstrating a $7.7 \mathrm{~cm}$-sized abscess in the $55 / 8$ segment of the liver. Percutaneous drainage was performed, and the culture result from the drainage came out as Rhizopus microsporus.

Results: Liposomal amphotericin B was administered intravenously at a dose of $5 \mathrm{mg} / \mathrm{kg}$ daily, and serial CTs were performed. Since the antifungal agent was initiated, the size of the lesions continuously decreased down to $5.8 \mathrm{~cm}$; however, there was no significant change thereafter. Accordingly, we decided to perform surgical resection for the lesion. Following approximately 3 months of antifungal therapy, the right anterior sectionectomy of the liver was performed. The antifungal agent was continuously used for 3 months after surgery. Kidney function was well preserved and there was no evidence of recurrences until 4 months after liver resection.

Conclusions: Optimal treatment strategies for mucormycosis in a renal transplant recipient have not yet been defined. In this case, even though, we were able to minimize the extent of liver resection by using an antifungal agent for a long period of time and successfully treat mucormycosis without damage of transplanted kidney. Early surgical intervention for the lesion could be a more valid option of treatment to shorten the duration of the disease and reduce medical expense.

Corresponding author: Cheol Woong Jung

E-mail: cwjung@korea.ac.kr

(c) The Korean Society for Transplantation

This is an Open Access article distributed under the terms of the Creative Commons Attribution Non-Commercial License (http://creativecommons.org/licenses/by-nc/4.0/) which permits unrestricted non-commercial use, distribution, and reproduction in any medium, provided the original work is properly cited. 